\title{
Performance of Reduced Titanium Oxide and Boron Doped Diamond as anodes in hyperthermophilic bioelectrochemical systems
}

\author{
Laura Malavola ${ }^{1}$, Silvia Franz $^{1 *}$, Massimiliano Bestetti ${ }^{1}$, Nunzia Esercizio $^{2}$, Giuliana D’Ippolito ${ }^{2}$, Pierangela Cristiani $^{3}$
}

${ }^{1}$ Politecnico di Milano, Department of Chemistry Materials and Chemical Engineering, 20131 Milano, Italy

${ }^{2}$ Institute of Biomolecular Chemistry (ICB), National Research Council (CNR), Pozzuoli, 80078, Na, Italy

${ }^{3}$ Ricerca sul Sistema Energetico - RSE S.p.A., 20134 Milano, Italy

\begin{abstract}
This work investigates Reduced Titanium Oxide (RTO) in comparison with Carbon Cloth (CC) and commercial Boron Doped Diamond (BDD) as anodes in hyperthermophilic bioelectrochemical systems operating at $80^{\circ} \mathrm{C}$ by Thermotoga neapolitana. Two samples of RTO were synthesized by plasma electrolytic oxidation (PEO) of titanium plates and subsequent electrochemical reduction. Electrochemical performance of CC, BDD, and RTO are tested by performing cyclic voltammetry in the anodic region ( 0 $1 \mathrm{~V}, 50 \mathrm{mV} / \mathrm{s}$ ), in abiotic and biotic conditions. The surface of colonized materials is observed by SEM microscopy. Results show that bacteria fast settle on all tested material, significantly affecting their electrochemical conductivity. The integration of voltammetric cycles reveals that biofilm generates capacitive effects on the anodic surfaces, particularly evident in RTO, less in CC and absent in BDD. Charge densities provided by capacitive response of RTO and CC are of the order of 5.58 and $0.77 \mathrm{mC} / \mathrm{cm}^{2}$, respectively.
\end{abstract}

\section{Introduction}

Thermotoga neapolitana are hyperthermophilic anaerobic bacteria, which produce hydrogen and lactate by metabolizing glucose. In principle, they can be exploited to catalyze the hydrogen evolution reaction in a microbial electrolytic cell, improving the overall yield of the system, as recently demonstrated [1]. Thermophilic systems are convenient to enhance the kinetics of the reactions involved and to promote the selectivity of the process, since other microbial strains, including pathogenic ones, are not allowed to grow. Previous works [2] found a great affinity of T.neapolitana to form biofilm on different materials. On the other hand, the effect of an oxidative polarization is supposed to excessively disturb the bacteria metabolism, due to the formation of reactive oxygen species (ROS). Furthermore, the electrochemical behavior of the electrode is modified when the bacterial colony attaches to its surface [1]. Carbon cloth is extensively used as electrode material for bioelectrochemical systems, since it provides very high surface area and porosity, as well as good conductivity, mechanical strength and flexibility. Among metals, the most used one is stainless steel, due to lower cost. Also copper and nickel have been investigated, leading to good performances, despite copper and nickel ions can be poisonous for microorganisms [3]. Stainless steel cathodes are commonly used, providing good catalytic effect when a wide surface area is available, but corrosion resistance should be improved [4]. On the other hand, bacteria reveal to be good catalysts for the cathodic reaction, enhancing the hydrogen production rate, without the use of metals [5].

The objective of this work is to investigate the change of the electrochemical properties of different types of anodes, and in particular Reduced titanium oxides (RTO), colonized by Thermotoga Neapolitana.

Reduced titanium oxides (RTO) were prepared and tested in comparison with a commercial Boron Doped Diamond (BDD) and carbon cloth (CC) in a double chamber electrochemical system. BDD is an inert anode, that is it does not participate in the reaction. It is a p-doped diamond that exhibits chemical inertness, corrosion resistance, extremely high hardness, thermal conductivity and good charge carrier mobility. With an overpotential of more than $1 \mathrm{~V}$ for oxygen evolution reaction (OER), it is able to oxidize the majority of organic compounds. Therefore, it allows the formation of oxidants on its surface that in turn will react with the organic matter [6], [7]. The main drawbacks of this material are certainly the high cost and the complex production process, that strongly limit its application on a large scale.

In this work, the performance of the BDD is used as a reference.

Cyclic voltammetry was performed before and after the colonization for each material. After the experiment, the surface of the electrodes was analyzed through SEM imaging. The results are here presented and discussed.

\section{MATERIALS AND METHODS}

\subsection{Reduced Titanium Oxide electrode preparation}

The reduced titanium oxides (RTO) were produced in three steps: starting from a c.p. titanium (Ti) plate, titanium dioxide $\left(\mathrm{TiO}_{2}\right)$ is obtained by means of plasma electrolytic oxidation (PEO), using two titanium meshes as counter electrodes and $1 \mathrm{~L}$ of $1.5 \mathrm{M} \mathrm{H}_{2} \mathrm{SO}_{4}$ as electrolyte. The operation was performed at $0^{\circ} \mathrm{C}$, under a voltage of $150 \mathrm{~V}$ and maximum current of $10 \mathrm{~A}$ for 5'. Thereafter, the samples were heat treated at $450^{\circ} \mathrm{C}$ for $3 \mathrm{~h}$

\footnotetext{
$\overline{\text { * Corresponding author: Silvia.Franz@ }}$ polimi.it
} 
in air environment. The material was then reduced through electrochemical treatment: the specimen was exposed at a current density of $-10 \mathrm{~mA} / \mathrm{cm}^{2}$ for $10 \mathrm{~min}$, using a platinum $(\mathrm{Pt})$ wire as counter electrode. The operation was carried out at $0^{\circ} \mathrm{C}$ in $1 \mathrm{~L}$ of $1.5 \mathrm{M} \mathrm{H}_{2} \mathrm{SO}_{4}$. This procedure, among four different methodologies, proved to lead to the best performing material. The four samples were synthesized through PEO, then post treated as follows:
A: no post treatment
$\mathrm{B}$ : thermal post-treatment in air at $450^{\circ} \mathrm{C}$ for $3 \mathrm{~h}$
$\mathrm{C}$ : thermal post-treatment as $\mathrm{B}$ and subsequent electrochemical reduction (as described)
D: thermal post-treatment and reduction, as $\mathrm{C}$, and, subsequently, de-hydrogenation in $\mathrm{Ar}$ environment at $400^{\circ} \mathrm{C}$ for $1 \mathrm{~h}$.

The samples were characterized by means of cyclic voltammetry $\mathrm{CV}$ (potential interval $-1.5-3 \mathrm{~V}$ vs $\mathrm{SCE}$, scan rate $20 \mathrm{mV} / \mathrm{s}$ ) and potentiostatic chronoamperometry (2.5 $\mathrm{V}$ vs SCE, $20 \mathrm{~min}$ ), carried out in $0.5 \mathrm{M} \mathrm{Na}_{2} \mathrm{SO}_{4}$ under dark conditions.

\subsection{Media and Inoculum}

$250 \mathrm{ml}$ of culture broth ATCC 1977 for anaerobic microorganisms, with trace element solution (DSM medium 141) and $5 \mathrm{~g} / \mathrm{l}$ of glucose was used as media. A stream of $\mathrm{CO}_{2}$ gas was sparged in the culture to create anaerobic condition, until the resazurin in the solution was colorless, as described for previous tests [1]. An aliquot of $7 \mathrm{ml}$ of pre-acclimate batch culture (at $80{ }^{\circ} \mathrm{C}$ for 1 day) were inoculated in each electrochemical bioreactor at the beginning of the test. All transfers and sampling of cultures were performed with sterile syringes and needles.

Thermotoga neapolitana subsp. capnolactica (DSM33033), a lab strain derived from T. neapolitana DSMZ 4359T [8] was tested.

\subsection{Electrochemical tests}

The electrochemical bioreactor was operated applying a voltage between the electrodes of $1.5 \pm 0.1 \mathrm{~V}$.

In order to compare the behavior of the anodic materials, four cycles of cyclic voltammetry were performed in the anodic region $0-1 \mathrm{~V}$ vs $\mathrm{Ag} / \mathrm{AgCl}(50 \mathrm{mV} / \mathrm{s})$ on the anodes, using a stainless-steel wire as counter electrode inserted in the anodic compartment. Measurements were repeated in abiotic and biotic conditions, $24 \mathrm{~h}$ after the inoculum of T. neapolitana.

The evaluation of capacitive effects generated by the biofilm has been derived from the integration of the $4^{\text {th }}$ cycle of current density curves. For each material, the charge density exchanged through anodic current in abiotic conditions is subtracted from the charge density exchanged through anodic current in biotic conditions. The difference is compared to the charge density exchanged through cathodic current in biotic conditions, hence the capacitive contribution is estimated.

\subsection{SEM analyses}

After the test, electrodes were dried 15 minutes in the oven at $80^{\circ} \mathrm{C}$ and stored at $4{ }^{\circ} \mathrm{C}$ until the analysis at Scanning Electron Microscopy (SEM). The used instrumentation is: Tescan Mira 3 - SEM field emission. The working voltage is $20 \mathrm{KV}$.

\section{RESULTS}

\subsection{Synthesis of reduced titanium oxide}

Through PEO a porous $\mathrm{TiO}_{2}$ coating of about $3 \mu \mathrm{m}$ is obtained, that appears light grey in color [9].

After the electrochemical reduction step the color shifts to light blue, in agreement with the reduction of band gap [10]. This is a first proof of enhanced conductivity with respect to the stoichiometric $\mathrm{TiO}_{2}$.

$\mathrm{CV}$ and chronoamperometry reveal that the electrochemically reduced sample $(\mathrm{C})$ is the best performing in terms of conductivity (Table 1). Indeed, A and D samples appear too resistive, B appears promising in the $\mathrm{CV}$, but the chronoamperometry shows a low current density at this voltage.

Hereafter, it is decided to proceed with the synthesis of the material $\mathrm{C}$ to be used in the bioelectrochemical system.

Table 1. Initial $\left(i_{0}\right)$ and final $\left(i_{1200}\right)$ current density and overpotential for OER $(\eta)$ of the synthesized materials and commercial BDD.

\begin{tabular}{|c|c|c|c|}
\hline & $\mathrm{i}_{0}\left(\mu \mathrm{A} / \mathrm{cm}^{2}\right)$ & $\mathrm{i}_{1200}\left(\mu \mathrm{A} / \mathrm{cm}^{2}\right)$ & $\eta(\mathrm{V})$ \\
\hline $\mathrm{A}$ & 143 & 7 & $3-5,8$ \\
\hline $\mathrm{B}$ & 0,9 & 0,4 & $1,9-2$ \\
\hline $\mathrm{C}$ & 56 & 14 & $1,8-1,9$ \\
\hline $\mathrm{D}$ & 0,9 & 0,1 & $4,1-5,5$ \\
\hline $\mathrm{BDD}$ & 1760 & 487 & 1,6 \\
\hline
\end{tabular}

\subsection{Cyclic voltammetry in culture medium}

In figure 1 , the $4^{\text {th }}$ cycle obtained in abiotic and biotic medium for each material is shown (1a: CC; 1b: BDD; 1c: RTO).

In the abiotic environment, after the inversion of potential scan, it appears that Ti-based electrodes continue some oxidation reactions forming oxidant species, while boron doped diamond presents a current density almost zero. We assume that the organic species attached to the electrode oxidized themselves and the glucose present in solution.

Carbon cloth electrode shows a wider capacitive effect compared to BDD and RTO. The onset of OER is definitely higher for BDD than for the other materials, as expected.

After the inoculum, instead, the curve obtained on RTO is much wider than for $\mathrm{CC}$, and this behavioral variation is not detected on BDD. The curve is integrated to obtain the charge density: the difference between the resulting 
value in biotic and in abiotic medium is an estimation of the capacitive effect arisen by microbial growth. For RTO, a contribution of $5.58 \mathrm{mC} / \mathrm{cm}^{2}$ is calculated, while on $\mathrm{CC}$ it was of $0.77 \mathrm{mC} / \mathrm{cm}^{2}$. On BDD no capacitive effect is observed, indeed, only an increase in current density is measured.
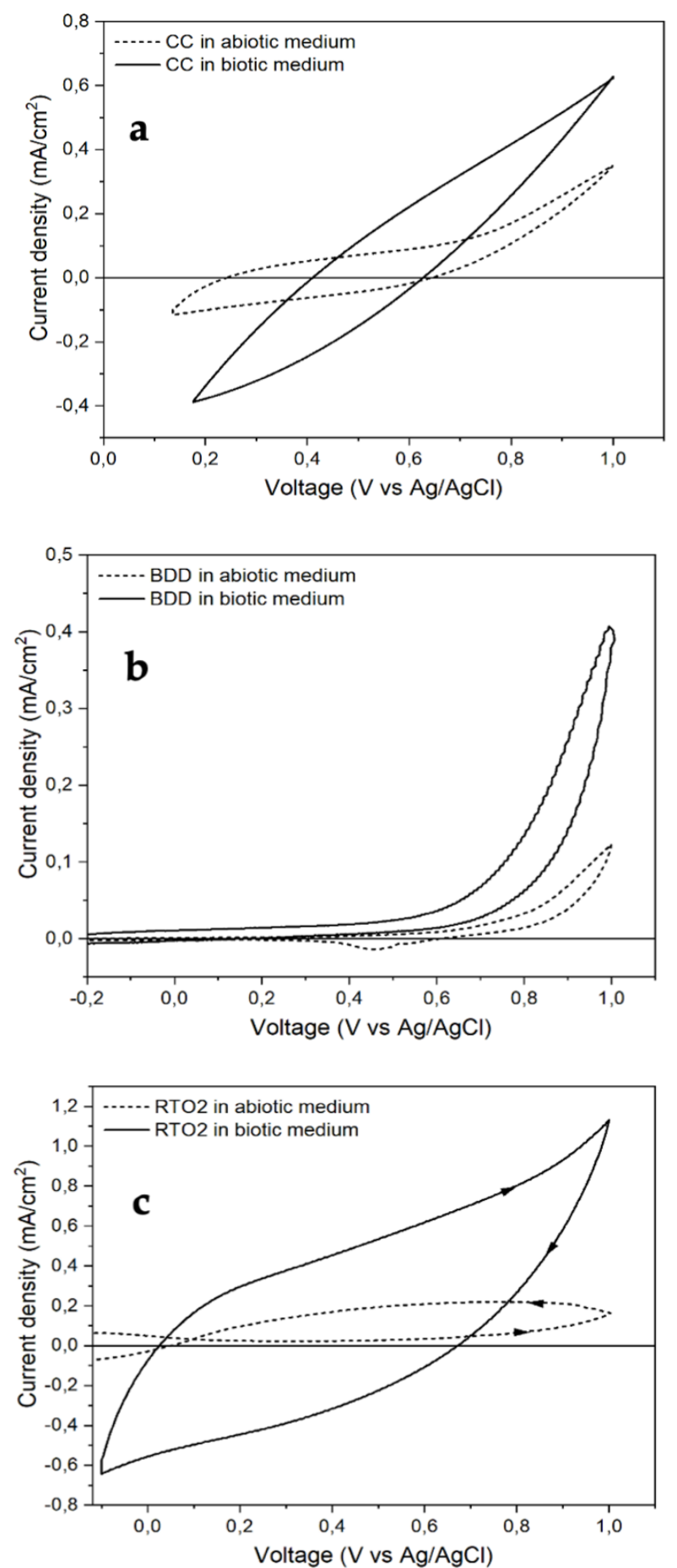

Fig. 1. Graphics of the $4^{\text {th }}$ cycle of voltammetry obtained in abiotic and biotic medium for each material (1a: CC; 1b: BDD; 1c: RTO).

\subsection{SEM images of the electrodes}

In figure $2 \mathrm{SEM}$ images of CC-PVdF1, C3, C1, RTO2, RTO1, BDD3 and A1 are reported.

SEM images of the cathodes (fig. 2.a-c) reveal that the fibers are covered by biofilm, meaning that bacteria are not only living in the bulk of the solution, but also on the electrode surface. On C3, that was subjected to a high circulating current, bacterial cells are not clearly visible in the biofilm appears different from the normal case: it is assumed that bacteria were damaged by the electrical stress, or that microorganisms detached, leaving biological matter on the surface.

From SEM images of the CC anode (fig. 2.i), it can be noticed that here bacteria have completely colonized the fibers. It is confirmed that $T$. neapolitana live on the anodic surface, thus confirming that their affinity with positive polarization is strong.

SEM images of BDD electrodes (fig. 2.g-h) reveal a uniform layer of biofilm adherent to the surface, and the typical aspect of BDD is hindered.

The inspection over RTO1 and RTO2 revealed that on both electrodes a biofilm is present, hence the edges of the porous structure are surrounded by a film (figure 2.d vs 2.e). Furthermore, in the case of RTO1 the biofilm formed was so thick $(\approx 250 \mathrm{~nm})$ that it cracked, as it can be seen in figure 2.f. Cells were not easy to detect, so that we assume bacteria were damaged by the high current circulating during the experiment.

On RTO2, the porous morphology of reduced $\mathrm{TiO} 2$ is observable, covered by a veil of biofilm.

\section{DISCUSSION}

Cyclic voltammetry in absence of bacteria confirm that BDD outperforms the other materials in terms of overpotential for OER, although RTO shows itself a good response.

The behavior of Reduced Titanium Oxide changes significantly, basing on whether bacteria are present or not: after the inoculum the material shows a capacitive behavior that was not detected before $\left(5.58 \mathrm{mC} / \mathrm{cm}^{2}\right)$. The capacitive effect is attributed to the biofilm formation, since hyperthermophilic bacteria $T$. neapolitana possess a toga rich in ionic species that improve the resistance to high temperatures [11]. The increased number of ionic species can actually enhance the conductivity of a surface, improving the performances of current exchange. The presence of hyperthermophilic $T$. neapolitana is detected by means of SEM on all anodic materials, meaning that in principle all of them are feasible for the application in bioelectrochemical systems, and bacteria are attracted by their positive polarization. However, it is probable that excessively high currents damage the microorganisms on RTO, while the biofilm, rich in ionic species, stays adherent to the negatively polarized surface. 

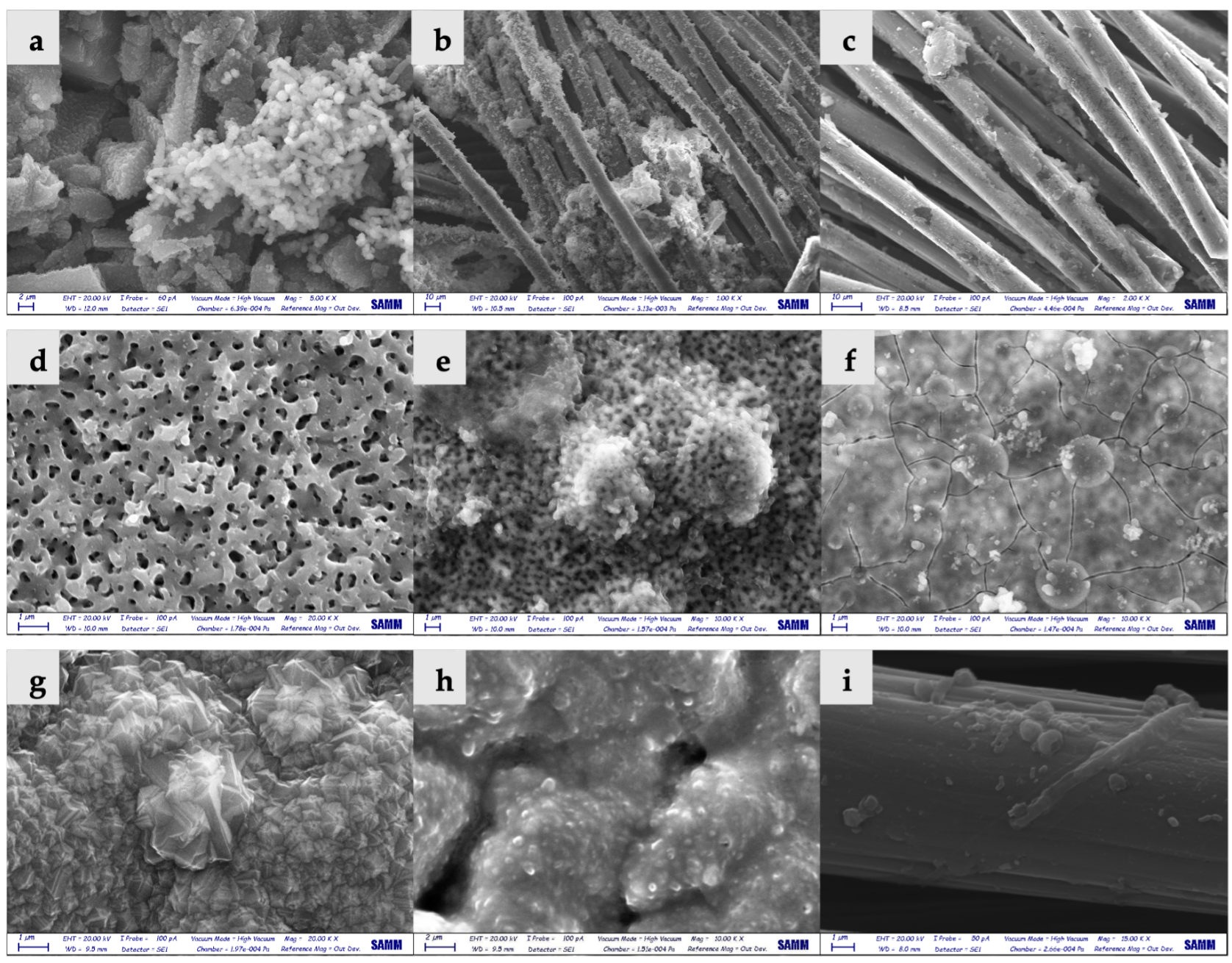

Figure 2. SEM images of cathodes: a) CC-PVdF1, b) C3 and c) C1; SEM images of anodes: d) RTO as prepared, e) RTO2, f) RTO1, g) BDD as purchased, h) BDD3, i) A1 (a fiber of carbon cloth).

Two hypotheses have been proposed on the nature of the capacitive effects of RTO and CC: either bacteria are "oxidized" on the surface, thus damaged by current circulating, or charges are simply transferred through the ionic species present in the biofilm. The latter option would be of great relevance: Thermotoga neapolitana strongly interact with the synthesized material, enhancing its conductivity. This would mean that RTO can be investigated more in depth even in single-chamber systems, where the damaging of cells cannot be an acceptable phenomenon. On the other hand, a sort of "cleaning" effect on the colonized electrode might be beneficial if a turn-over of active cells is preferred, for instance, to limit issues related to the mass transfer of nutrients at the interface between solid and biofilm.

The capacitive response recognized on RTO is present with lower effect on $\mathrm{CC}\left(0.77 \mathrm{mC} / \mathrm{cm}^{2}\right)$, and not observed at all on BDD, where only the faradaic effect of the reaction emerges from cyclic voltammetry. The comparison between RTO and CC suggests that the former could be used in high voltage regime, without the risk of corrosion, encountered on the latter.

If, instead, bacteria damaged by current circulating might reach unacceptable level, boron doped diamond would be the best performing material to be implemented in the bioelectrochemical system, due to the absence of capacitive behavior even if colonized by bacteria.

\section{CONCLUSIONS}

Reduced titanium oxide (RTO) has been synthesized by means of plasma electrolytic oxidation (PEO), thermal treatment and subsequent electrochemical reduction. This procedure is certainly convenient in terms of time and costs.

Comparisons have been made between RTO, BDD and CC using the ATCC 1977 medium as electrolyte.

The best material can be chosen basing on different criteria: BDD is the best performing to maintain anaerobic conditions, moreover it does not involve the biofilm in the exchange of charges with the solution. RTO could be used to enhance conductivity of the system, even further if microorganisms are present on its surface.

The tested materials offer the possibility of choosing the best solution: a material that guarantees the absence of oxygen evolution on the anode while oxidizing the

* Corresponding author: Silvia.Franz@polimi.it 
organic matter (BDD), or a material which electrical performance is positively affected by the presence of microorganisms in a high current regime, and that is definitely more economic (RTO).

Further investigations on the used materials and their interaction with hydrogen-producing bacteria $T$. neapolitana are required to elucidate the effect on bacteria metabolism.

\section{Acknowledgements}

This work was financed by the Research Fund for the Italian Electrical System in compliance with the Decree of March, 19th 2018.

\section{References}

[1] G. d'Ippolito et al., "Electrostimulation of hyperthermophile Thermotoga neapolitana cultures," Bioresour. Technol., vol. 319, p. 124078, Jan. (2021).

[2] G. Squadrito et al., "Hyperthermiphile biofilms of Thermotoga neapolitana on different materials and electrostimulated: SEM micrographs and chemical data of the glucose fermentation in electrochemical reactors, " Data $\mathrm{Br}$., vol. 33, p. 106403, (2020).

[3] A. Baudler, I. Schmidt, M. Langner, A. Greiner, and U. Schröder, "Does it have to be carbon? Metal anodes in microbial fuel cells and related bioelectrochemical systems, ,
Energy Environ. Sci., vol. 8, no. 7, (2015).

[4] P. Dange et al., "Recent developments in microbial electrolysis cell-based biohydrogen production utilizing wastewater as a feedstock, " Sustainability (Switzerland), vol. 13, no. 16. (2021).

[5] R. A. Rozendal, A. W. Jeremiasse, H. V. M. Hamelers, and C. J. N. Buisman, "Hydrogen production with a microbial biocathode," Environ. Sci. Technol., vol. 42, no. 2, (2008).

[6] E. Brillas and C. A. Martínez-Huitle, Synthetic Diamond Films: Preparation, Electrochemistry, Characterization, and Applications. (2011).

[7] M. Panizza and G. Cerisola, "Application of diamond electrodes to electrochemical processes," Electrochim. Acta, vol. 51, no. 2, pp. 191-199, (2005).

[8] L. Dipasquale, G. D’Ippolito, and A. Fontana, “Capnophilic lactic fermentation and hydrogen synthesis by Thermotoga neapolitana: An unexpected deviation from the dark fermentation model, ” Int. J. Hydrogen Energy, vol. 39, no. 10, pp. 4857-4862, Mar. (2014).

[9] S. Franz, H. Arab, G. L. Chiarello, M. Bestetti, and E. Selli, "Single-Step Preparation of Large Area TiO 2

Photoelectrodes for Water Splitting, ” Adv. Energy Mater., vol. 10, no. 23, p. 2000652, Jun. (2020).

[10] C. Hauf, R. Kniep, and G. Pfaff, "Preparation of various titanium suboxide powders by reduction of TiO2 with silicon, ” J. Mater. Sci., vol. 34, no. 6, pp. 1287-1292, (1999).

[11] G. Auerbach et al., "Lactate dehydrogenase from the hyperthermophilic bacterium Thermotoga maritima: the crystal structure at 2.1 a resolution reveals strategies for intrinsic protein stabilization," Structure, vol. 6, no. 6, pp 769-781, Jun. (1998). 